\title{
Emission, réception et réflexion d'ondes à la surface d'un milieu anisotrope
}

\author{
M. Planat, J. M. Hamet et P. Schiavone \\ Laboratoire de Physique et Métrologie des Oscillateurs du CNRS, \\ associé à l'Université de Franche-Comté-Besançon, 32, avenue de l'Observatoire, 25000 Besançon, France
}

(Reçu le 29 novembre 1984, révisé le 5 février 1985, accepté le 8 février 1985)

\begin{abstract}
Résumé. - Nous étudions en premier lieu les ondes de surface et de volume émises et détectées par un transducteur métallique interdigité déposé à la surface d'un milieu anisotrope faiblement piézoélectrique. Il est montré qu'en régime harmonique le réseau émet non seulement l'onde de Rayleigh (ou la pseudo-onde de Rayleigh), mais qu'il rayonne aussi trois ondes de volume. Ces conclusions sont confirmées par les mesures effectuées à l'aide de lignes à retard et de résonateurs à quartz.

La méthode est ensuite utilisée pour étudier la réflexion d'une onde de Rayleigh sous un sillon gravé peu profond.

Abstract. - Surface and bulk acoustic waves excited and detected by a metallic interdigital transducer deposited on the surface of an anisotropic low piezoelectric medium are studied first. In the harmonic mode, the comb emits the Rayleigh wave (or the pseudo-Rayleigh wave) and also three bulk waves called " surface skimming bulk waves ». This result is compared to experiments on surface wave quartz delay lines and resonators.

The method is then applied to the reflexion of a Rayleigh wave under a low deep groove.
\end{abstract}

\section{Introduction.}

Depuis la première réalisation de White [1], les ondes de surface ont eu un essor considérable en électronique. Le dispositif de base, la ligne à retard, est constitué de deux peignes métalliques interdigités (Fig. 1), l'un pour l'excitation, l'autre pour la détection, déposés sur la surface d'un milieu piézoélectrique par photolithographie. Construit pour émettre des ondes de Rayleigh (leur fréquence $f$ est fixée par la périodicité $\lambda / 2=V_{\mathbf{R}} / 2 f$ où $V_{\mathbf{R}}$ est la vitesse de l'onde de Rayleigh), on a observé que le peigne émet également des ondes de volume $[2,3]$. Ces ondes de volume, dites rampantes sous la surface (surface skimming bulk waves ou SSBW) possèdent un certain nombre de caractéristiques avantageuses par comparaison avec les ondes de Rayleigh : comme elles se propagent plus vite, elles permettent une fréquence de fonctionnement plus élevée pour le même transducteur; elles ont de plus une meilleure stabilité en température et un "vieillissement" inférieur. Inversement, les ondes SSBW peuvent apparaître en parasites dans les filtres à large bande en créant des interférences avec l'onde de Rayleigh ou en augmentant

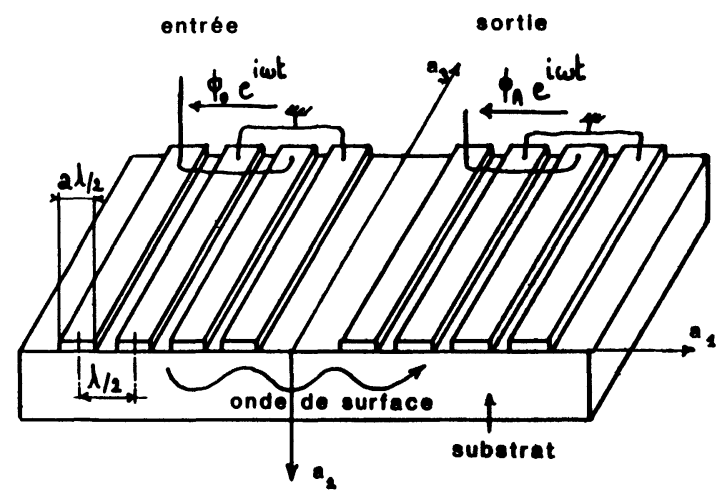

Fig. 1. - Schéma d'une ligne à retard à ondes de surface.

[Schematic of a surface acoustic wave delay line.]

les pertes d'insertion puisqu'elles peuvent être une source importante de perte d'énergie.

Le problème fondamental de l'excitation d'ondes de surface par un peigne a été étudié dans le cas d'un cristal faiblement piézoélectrique à symétrie hexagonale [4] ou trigonale [5]. Une autre approche [6] ne fait pas l'hypothèse des faibles couplages mais requiert 
une procédure numérique implicite pour calculer la distribution des charges électriques sur les peignes. Nous avons présenté récemment l'étude de l'excitation d'ondes de Rayleigh dans les milieux anisotropes faiblement piézoélectriques [7]. Des expressions analytiques simples permettent de relier les tensions électriques sur les transducteurs à l'amplitude mécanique de l'onde, et d'en extraire les paramètres de dispersion de la ligne à retard.

Le modèle est étendu ici à l'émission des ondes de volume SSBW, puis à la réflexion des ondes de Rayleigh sous un sillon gravé faiblement profond. La philosophie de ce travail est déjà contenue dans le célèbre mémoire de $H$. Lamb [8].

\section{Emission et détection.}

Le dispositif à étudier est représenté figure 1. Les transducteurs sont supposés infiniment larges pour ne pas tenir compte des effets de diffraction et minces pour négliger les contraintes mécaniques de surface. Comme le coefficient de couplage piézoélectrique est faible, on sépare le problème en deux parties. On calcule d'abord le potentiel électrostatique créé à l'intérieur du milieu par l'application de la tension électrique $\phi_{0}$, en négligeant l'induction électrique due à la présence de l'onde. Puis, on résout les équations d'équilibre et les conditions aux limites avec au second membre les termes piézoélectriques connus.

2.1 Potentiel ÉleCtrostatiQue CRÉÉ PAR LE PEIGNe. - Le potentiel électrostatique $\Phi$ dû au réseau périodique d'entrée est donné par l'équation de Laplace

$$
\varepsilon_{i l} \Phi_{, l i}=0,
$$

où les $\varepsilon$ sont les constantes diélectriques et , $i$ représente la dérivée partielle par rapport à la coordonnée d'espace $a_{i}$. Les conditions aux limites expriment l'annulation de la composante tangentielle du champ électrique sur les électrodes et la continuité du déplacement électrique normal entre les électrodes. Dans le cas d'un transducteur très long, de largeur $W$ et possédant $N_{1}$ paires de doigts, on montre [9] que la capacité $C_{1}$ est donnée par la relation

$$
C_{1}=N_{1} W\left(\varepsilon_{0}+\varepsilon_{22} \varepsilon_{\mathrm{t}}\right) K(s) / K\left(s^{\prime}\right)
$$

où $s=\sin a \pi / 2$ et $K(s), K\left(s^{\prime}\right)$ sont les intégrales elliptiques de première espèce pour les modules $s$ et $s^{\prime}=\left(1-s^{2}\right)^{1 / 2}$. L'espacement entre deux doigts est $\lambda / 2$ et $a$ est la largeur relative des doigts par rapport à l'espacement; $\varepsilon_{0}$ est la constante diélectrique. L'anisotropie du milieu apparaît dans la constante sans dimension $\varepsilon_{\mathrm{t}}=\left(\varepsilon_{11} \varepsilon_{22}-\varepsilon_{12}^{2}\right)^{1 / 2} / \varepsilon_{22}$.

2.2 DÉPlaCEMENTS MÉCANIQUES. - En régime harmonique, à la fréquence $\omega$, les déplacements mécaniques $u_{j}$ s'obtiennent à partir des conditions d'équi- libre et des conditions aux limites qui expriment l'annulation de la contrainte en $a_{2}=0$ et du déplacement en $a_{2}=\infty$ :

$$
\begin{gathered}
\rho_{0} \omega^{2} u_{j}+C_{i j k l} u_{k, l i}=-e_{l i j} \phi_{, l i} \\
C_{2 j k l} u_{k, l}=-e_{l 2 j} \phi_{, l} \text { si } a_{2}=0 \\
u_{j}=0 \text { si } a_{2}=\infty,
\end{gathered}
$$

où $\rho_{0}$ est la masse volumique et $C$ et $e$ sont les constantes élastiques et piézoélectriques du substrat.

Introduisant le nombre d'ondes $k$, le système (3)-(4) est résolu à l'aide de la transformée de Fourier $\hat{X}$ due au champ $\mathrm{X}$ d'après la relation :

$$
\hat{X}\left(k, a_{2}\right)=1 / \sqrt{2 \pi} \int_{-\infty}^{+\infty} X\left(a_{1}, a_{2}\right) e^{i k a_{1}} \mathrm{~d} a_{1} .
$$

On en déduit [7] la transformée de Fourier $\hat{u}_{j} d u$ déplacement mécanique sous la forme :

$$
\hat{u}_{j}=t_{j} \hat{\phi}+\sum_{r=1}^{3} A_{r} a_{j}^{(r)} \mathrm{e}^{-i k n_{2}^{(r)} a_{2}} .
$$

Le premier terme de cette expression est la solution particulière pour la transformée de Fourier de l'équation de propagation (3). Le second terme est une solution générale de la transformée de Fourier de (3) sans second membre qui satisfait également à la condition aux limites, transformée de Fourier de (4). Afin de pouvoir également vérifier la condition (5), 3 modes de surface de coefficient de pénétration $n_{2}^{(r)}$ sont retenus, ceux dont la partie imaginaire est négative.

Les coefficients $t_{j}, A_{r}$ et $a_{j}^{(r)}$ sont donnés à la référence [7].

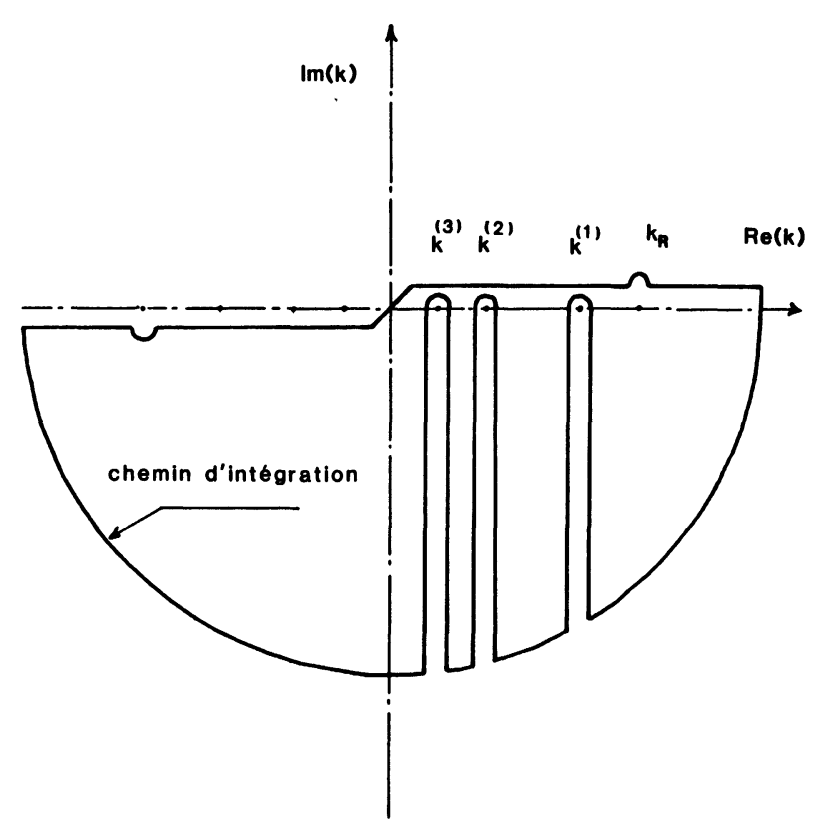

Fig. 2. - Chemin d'intégration dans le plan complexe des nombres d'ondes pour le calcul du déplacement mécanique.

[Integration path in the complex plane of wave-numbers to calculate the mechanical displacement.] 
Les déplacements s'obtiennent par transformée de Fourier inverse :

$$
\begin{array}{r}
u_{j}\left(a_{1}, a_{2}\right)=\frac{1}{\sqrt{2 \pi}} \int_{-\infty}^{+\infty}\left(\sum_{r=1}^{3} \frac{B_{r}}{\Delta} a_{j}^{(r)} \mathrm{e}^{-i k n_{2}^{(r)} a_{2}}+\right. \\
\left.+t_{j} \exp \left[\left(-\varepsilon_{r}+\frac{i \varepsilon_{12}}{\varepsilon_{22}}\right) k a_{2}\right]\right) \hat{\phi}_{0} \mathrm{e}^{-i k a_{1}} \mathrm{~d} k,
\end{array}
$$

où $\hat{\phi}_{0}(k)=\hat{\phi}(k, 0) ; B_{r}, \Delta$ et $a_{j}^{(r)}$ sont fonction du nombre d'ondes d'intégration $k$ mais ne peuvent pas être exprimés explicitement dans le cas général des milieux anisotropes. $\Delta$ est le déterminant de la condition aux limites d'inconnue $A_{r}$.

Le calcul de l'intégrale (8) est effectué en utilisant une variable d'intégration $k$ complexe. Pour le cas d'ondes se propageant dans la direction $\mathrm{O} a_{1}$, le contour d'intégration est représenté figure 2 .

Deux types d'ondes peuvent être émises : les ondes de Rayleigh et les ondes SSBW.

2.3 ONDE De RAYLeigh. - L'onde de Rayleigh (ou la pseudo-onde de Rayleigh [10]) correspond à un pôle $k_{\mathrm{R}}=\omega / V_{\mathrm{R}}$ de l'intégrant de (8). Le déplacement correspondant peut être obtenu simplement en calculant le résidu du pôle. L'amplitude normalisée $U_{1}$ de l'onde a la forme :

$$
U_{1}=-\pi \phi_{0} N_{1} B_{1} F\left(k_{\mathrm{R}}\right) a_{1}^{(1)} / K\left(s^{\prime}\right) V_{\mathrm{R}}\left(\frac{\mathrm{d} \Delta}{\mathrm{d} V}\right)_{v_{\mathrm{R}}},
$$

où $F\left(k_{\mathrm{R}}\right)$ a l'allure de fonctions en $\sin x / x$ et où $a_{1}^{(1)}=1 \mathrm{~m}^{2}$.

Du flux de puissance $\mathfrak{T}$ rayonné dans chaque direction et calculé d'après (9), on extrait l'admittance de rayonnement $G_{a}$ du transducteur donnée par :

$$
2 \mathfrak{T}=\frac{1}{2} \phi_{0}^{2} G_{a}(\omega),
$$

puis sa susceptance [11] :

$$
B_{a}(\omega)=-\frac{1}{\pi} G_{a}(\omega) * \frac{1}{\omega},
$$

où * représente le produit de convolution.

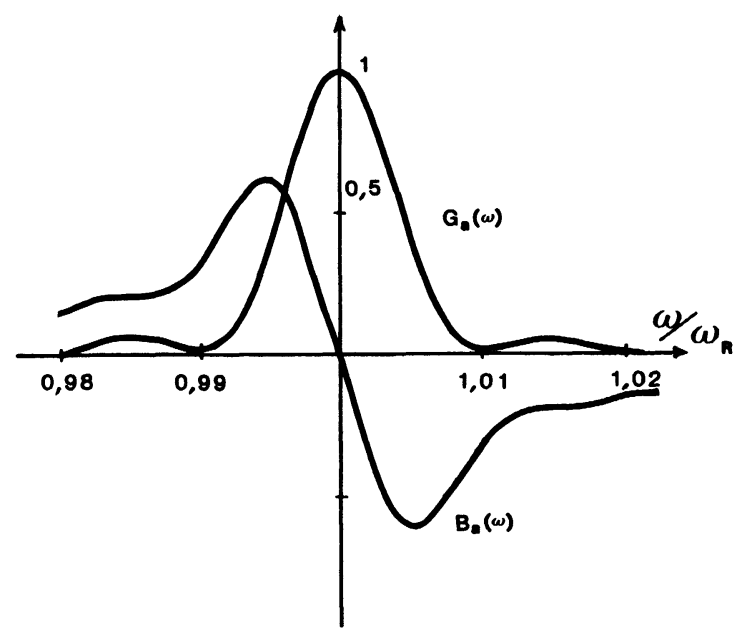

Fig. 3. - Admittance et susceptance de rayonnement du transducteur sous forme d'ondes de Rayleigh $\left(N_{1}=100\right.$; $a=0,5$; substrat de quartz de coupe Y).

[Radiation admittance and susceptance of the Rayleigh wave transducer $\left(N_{1}=100, a=0.5 ; \mathrm{Y}\right.$ cut quartz substrate).]

Leur allure est représentée figure 3 pour $N_{1}=100$ et $a=0,5$ dans le cas d'un substrat de quartz de coupe $Y$.

La détection des ondes de Rayleigh est analysée à la référence [7]; la tension détectée sur le transducteur de sortie de capacité $C_{2}$ et d'admittance $Y$ a la forme :

$$
\phi_{\mathrm{A}}=\frac{\pi}{K(s)} \phi_{\mathrm{s}} U_{1} \omega C_{2} / Y+i \omega C_{2},
$$

où $\phi_{\mathrm{s}}$ est uniquement fonction des constantes du milieu de propagation.

2.3.1 Résultats. - On a calculé les paramètres de transmission $S_{21}$ et de réflexion $S_{11}$ à la fréquence de synchronisme dans le cas de diverses coupes de quartz et comparé ces résultats à ceux mesurés sur un analyseur de réseau.

Si $Z_{1}, Z_{0}$ et $\alpha$ sont respectivement l'impédance du transducteur d'entrée, l'impédance caractéristique et

Tableau I. - Paramètres de dispersion pour des lignes à retard de quartz. Résultats calculés (C) et mesurés (M).

[Dispersion parameters for various quartz delay lines. Calculated (C) and measured (M) results.]

\begin{tabular}{|l|c|c|c|c|c|c|c|c|c|}
\hline \multicolumn{1}{|c|}{ Dispositif } & Fréquence & \multicolumn{2}{c|}{$S_{11}$} & \multicolumn{2}{c|}{$S_{21}$} & \multicolumn{2}{c|}{$S_{22}$} & \multicolumn{2}{|c|}{$S_{12}$} \\
& $(\mathrm{MHz})$ & $\mathrm{C}$ & $\mathrm{M}$ & $\mathrm{C}$ & $\mathrm{M}$ & $\mathrm{C}$ & $\mathrm{M}$ & $\mathrm{C}$ & $\mathrm{M}$ \\
\hline 1 (coupe ST) & 91,6 & $-1,2$ & $-2,0$ & $-13,5$ & $-12,8$ & $-2,7$ & $-4,2$ & $-13,6$ & $-12,8$ \\
\hline 2 (coupe ST $\left.+\Psi=36^{\circ}\right)$ & 92 & $-1,6$ & $-2,3$ & $-11,6$ & $-11,6$ & $-3,5$ & $-5,7$ & $-11,8$ & $-11,5$ \\
\hline 3 (coupe FST) & 99,6 & $-3,0$ & $-2,1$ & $-10,2$ & $-13,5$ & & & & \\
\hline 4 (coupe AT) & 300 & $-4,1$ & -14 & $-6,5$ & -10 & $-9,9$ & $-14,4$ & $-6,9$ & $-10,1$ \\
\hline
\end{tabular}


le rapport entre les tensions de sorties et d'entrée, les paramètres de réflexion $S_{11}$ et de transmission $S_{21}$ sont donnés par les relations :

$S_{11}=Z_{1}-Z_{0} / Z_{1}+Z_{0}$ et $S_{21}=2 \alpha Z_{1} / Z_{1}+Z_{0}$

où $1 / Z_{1}=G_{a}-j C_{1} \omega$ et $Z_{0}=50 \Omega$.

Les résultats sont donnés dans le tableau I pour 4 lignes à retard différentes. La première (1) est construite sur un substrat de quartz de coupe ST, avec $N_{1}=100$ paires de doigts (peigne d'entrée), $N_{2}=150$ (peigne de sortie), $W=2500 \mu \mathrm{m}, a=0,5$ et $\omega=6,28 \times 10^{8} \mathrm{~Hz}$. La seconde (2) utilise le même substrat, des peignes identiques, mais la direction de propagation est tournée de l'angle $\Psi=36^{\circ}$. La troisième est la coupe à double rotation $\operatorname{FST}\left(\theta=-41,5^{\circ}\right.$; $\phi=6,3^{\circ} ; \Psi=26^{\circ}$ ) et deux peignes identiques ont été utilisés $(N=150)$. Enfin la dernière ligne utilise un substrat de coupe AT avec $N_{1}=150 ; N_{2}=225$; $W=1000 \mu \mathrm{m}$ et $\omega=18,9 \times 10^{8} \mathrm{~Hz}$.

La comparaison avec les valeurs théoriques fournit un assez bon accord dans le cas des deux premières coupes. Dans la troisième, la présence des ondes de volume dans la bande passante modifie notablement le coefficient de transmission. Enfin pour la dernière coupe, les différences sont essentiellement dues à la non-uniformité de la structure du peigne et aux effets d'adaptation dans les connexions.

2.4 ONDES DE vOlumE SSBW. - Les ondes de volume sont émises aux points critiques de l'intégrant de (8). Le polynôme qui exprime les coefficients de pénétration $n_{2}$ est issu de la partie homogène de la transformée de Fourier de l'équation de propagation (3). Il est du $6^{\mathrm{e}}$ degré et possède le nombre d'ondes comme paramètre. Ses racines sont en général complexes et deux à deux conjuguées; elles correspondent à trois termes évanescents $(r=1,3)$ et à 3 termes physiquement inacceptables puisque leur amplitude s'amplifie indéfiniment vers l'intérieur du matériau. Lorsque pour certaines valeurs du nombre d'ondes $k$ la partie imaginaire de $n_{2}$ est nulle, on obtient une racine double du polynôme. Ceci se produit en général pour 3 valeurs $k^{(p)}$ où $p=1,3$. L'intégrant de (8) qui contient la fonction $n_{2}\left(k^{(p)}\right)$ n'est plus holomorphe en ce point critique puisqu'il $y$ possède une racine carrée. C'est pourquoi ces nombres d'ondes ont été exclus du contour d'intégration (Fig. 2) en pratiquant 3 coupures. Les ondes émises proviennent de la contribution des chemins d'intégration autour des coupures. Cette contribution peut être calculée en utilisant la méthode donnée par Ken'ichiro Yashiro [12]. Il est cependant plus simple de calculer l'effet produit à grande distance $R$ du transducteur dans une direction donnée $\xi\left(a_{1}=R\right.$ $\left.\sin \xi ; a_{2}=R \cos \xi\right)$ en utilisant la méthode de la phase stationnaire [13] :

Lorsque $\boldsymbol{R}$ tend vers l'infini, seul le premier terme dans l'expression (8) subsiste. De plus, l'exponentielle oscille très rapidement dans les régions où la phase varie de façon appréciable. Par conséquent, dans ces régions, l'intégrale (8) est nulle. Ne conservant que les valeurs $k=k^{(p)}(p=1,3)$ qui rendent la phase stationnaire, on obtient :

$$
\begin{aligned}
u_{j}=\sum_{p=1}^{3} \sum_{r=1}^{3} & \delta(r, p)\left[2 \pi / R\left|\frac{\mathrm{d}^{2} f^{(r)}}{\mathrm{d} k^{2}}\right|_{k=k(p)}\right]^{1 / 2} \\
& \times \exp \left[-i \frac{\pi}{4}\right] \mathrm{e}_{j}^{(r)}\left(k^{(p)}\right) \mathrm{e}^{i R f(r)\left(k^{(p)}\right)}
\end{aligned}
$$

avec :

$$
\begin{gathered}
\mathrm{e}_{j}^{(r)}(k)=\frac{1}{\sqrt{2 \pi}}\left[\frac{B_{r} a_{j}^{(r)} \hat{\phi}_{0}}{\Delta}\right](k) \\
f^{(r)}(k)=k\left[n_{2}^{(r)}(k) \cos \xi+\sin \xi\right] . \\
\frac{\mathrm{d}}{\mathrm{d} k}\left[k \operatorname{Re}\left(n_{2}^{(r)}(k)\right]_{k=k^{(p)}}=-\operatorname{tg} \xi,\right.
\end{gathered}
$$

où $\operatorname{Re}$ désigne la partie réelle et $\delta$ le symbole de Kronecker.

Comme à la surface du matériau, l'angle $\xi$ vaut $\pi / 2$, on voit qu'en un point de phase stationnaire, la partie réelle du coefficient de pénétration $n_{2}$ possède une pente verticale. En ce point, la partie imaginaire de $n_{2}$ est nulle, nous obtenons donc l'identité entre la position des points critiques et la position des points de phase stationnaire, ce qui est déjà admis implicitement dans les relations précédentes.

La relation (15) nous permet aussi de déterminer la valeur du nombre d'ondes en un point intérieur au volume situé sous l'incidence $\xi$. Elle exprime le contact entre la droite de pente - $\operatorname{tg} \xi$ et la fonction $g(k)=k \operatorname{Re}\left(n_{2}(k)\right)$ au point de phase stationnaire.

2.4.1 Résultats. - L'allure typique de la fonction $g(k)$ est représentée figure 4 pour le cas de quartz coupe AT, $\Psi=-90^{\circ}$ ( $\Psi$ représente la direction de propagation relativement à l'axe $\left.\mathrm{O} a_{1}\right)$. On observe 3 points critiques correspondant à 3 ondes SSBW se propageant à la surface du matériau avec les vitesses respectives 3743,5089 et $6156 \mathrm{~m} / \mathrm{s}$. On a aussi obtenu graphiquement à titre d'exemple la vitesse $V_{45}=3900 \mathrm{~m} / \mathrm{s}$ de la première onde lorsqu'elle se propage à l'incidence $\xi=45^{\circ}$.

La coupe AT $-90^{\circ}$, apparaît fréquemment dans la littérature à propos des ondes SSBW, mais seul le deuxième point critique est cité. La vitesse expérimentale $5000 \mathrm{~m} / \mathrm{s}$ est en bon accord avec nos calculs. Notons (relation (13)) que l'amplitude du déplacement est en liaison directe avec le rayon de courbure au point critique considéré; la figure 4 montre que celui-ci est considérablement plus élevé pour le deuxième point critique $(5089 \mathrm{~m} / \mathrm{s}$ que pour les 2 autres.

La vitesse de l'onde SSBW a été obtenue précédemment par Lee [14] en fonction du premier angle de rotation $\theta$ autour de l'axe $\mathrm{O} a_{1}$ en partant de la coupe $\mathrm{Y}$, la direction de propagation étant toujours $\Psi=-90^{\circ}$ (Fig. 5 : courbes en pointillés). 


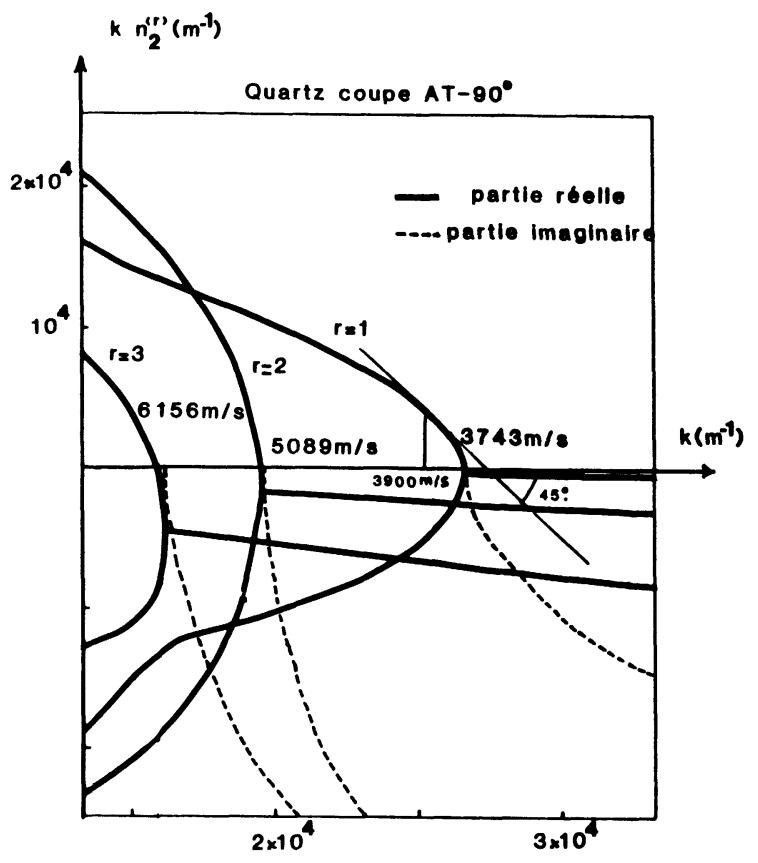

Fig. 4. - Evolution de la phase des 3 modes de surface $(r=1,3)$ en fonction du nombre d'ondes, dans le cas d'un substrat de quartz de coupe AT, à l'angle de propagation $\Psi=-90^{\circ}$.

[Phase of the three surface modes $(r=1.3)$ as a function of wave number (AT cut quartz substrate, propagation angle : $\left.\Psi=-90^{\circ}\right)$.]

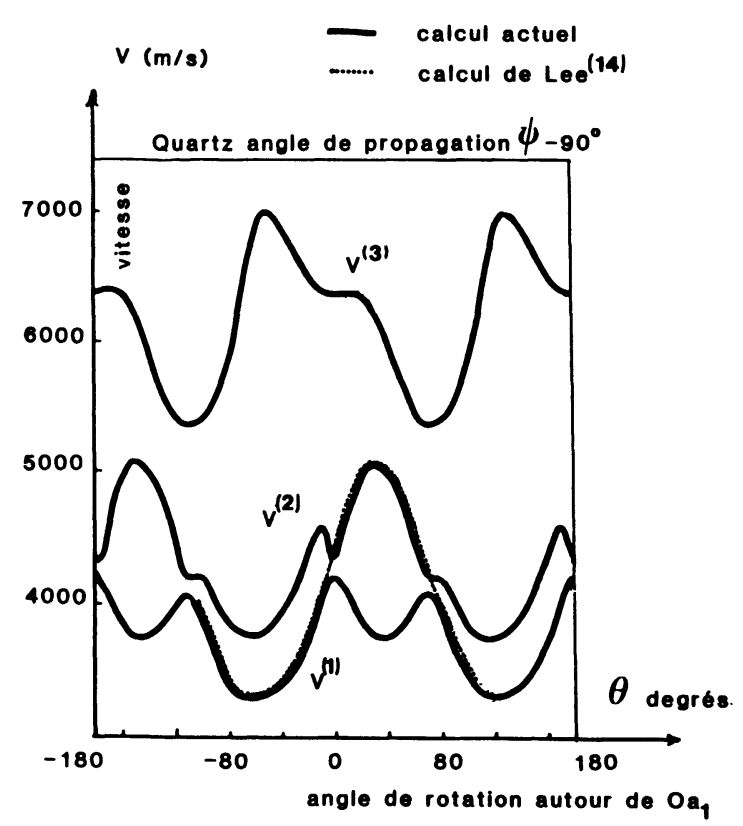

Fig. 5. - Vitesses des 3 ondes SSBW en fonction de l'angle de rotation $\theta$ autour de l'axe $\mathrm{O} a_{1}$ d'un substrat de quartz de coupe $Y$. Les courbes en pointillés proviennent du calcul effectué à la référence [14].

[SSBW velocities as a function of the rotation angle $\theta$ around the $\mathrm{O} a_{1}$ axis of a Y cut quartz substrate. Dotted curves come from the calculation of reference [14].]
De notre côté, nous avons déterminé la position des 3 points critiques pour les mêmes orientations cristallines; la figure 5 montre que les valeurs obtenues à la référence [14] proviennent de deux points critiques distincts.

Pour valider ce résultat nouveau de 3 points critiques, nous avons observé la courbe de réponse en phase et en amplitude d'une ligne à retard de coupe AT (Fig. 6). L'existence des deux points critiques aux vitesses les plus élevées, 5103 et $5723 \mathrm{~m} / \mathrm{s}$ est confirmée par la présence des deux raies aux fréquences 148,6 et $167 \mathrm{MHz}$. Le premier point critique est difficile à observer car il est inclus dans la bande passante de l'onde de Rayleigh représentée ici par le signal à 91,5 MHz.

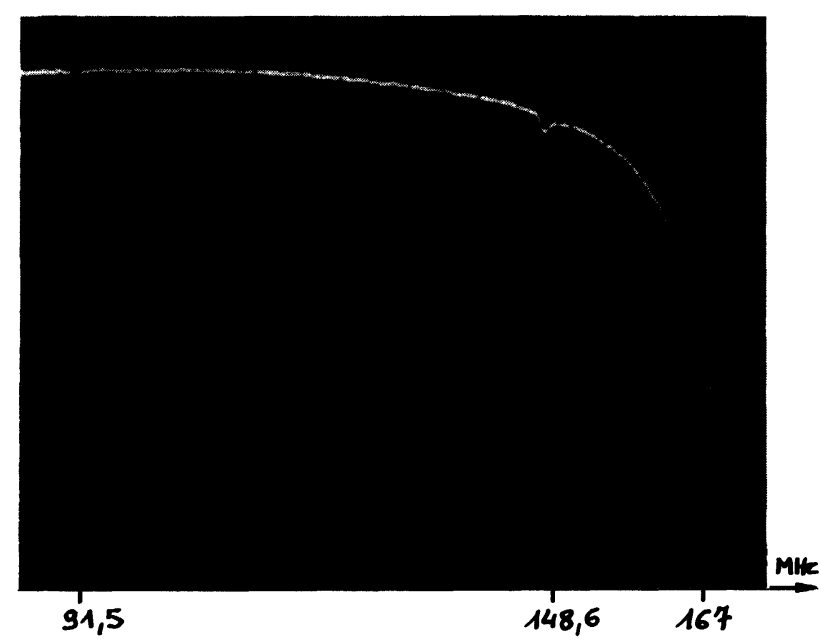

$$
\text { (a) } \quad \mathrm{S}_{11}(1 \mathrm{~dB} / \text { division) }
$$

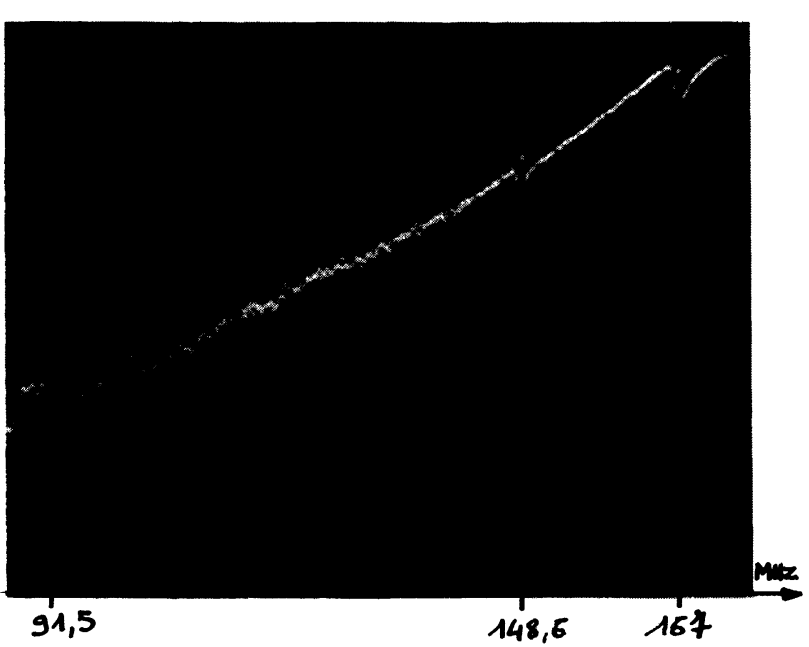

$$
\text { (b ) } \quad S_{21}(5 d B / \text { division) }
$$

Fig. 6. - Fonction de transfert d'une ligne à retard de quartz de coupe AT $(\lambda=34,4 \mu \mathrm{m})$. a) Coefficient de réflexion $S_{11}$, b) Coefficient de transmission $S_{22}$.

[Response curve of a AT cut quartz delay line $(\lambda=34.4 \mu \mathrm{m})$. a) Reflexion coefficient $S_{11}$, b) Transmission coefficient $S_{21}$.] 
La courbe de réponse d'un résonateur à quartz de coupe $\mathrm{Y}$ a également été observée (Fig. 7). Si les points critiques aux fréquences supérieures sont aisément obtenus, le premier point critique n'est pas distingable des lobes secondaires de l'onde de Rayleigh. Sa présence est cependant attestée par de nombreux phénomènes de parasitage dans les filtres et les capteurs de température à ondes de Rayleigh; comme l'interprétation est relativement indirecte, nous n'en parlerons pas ici.

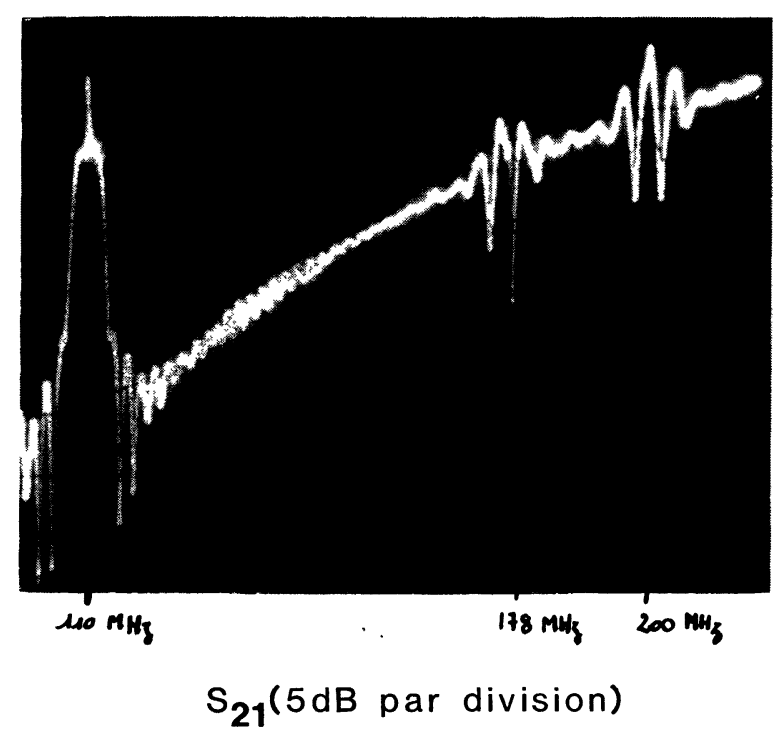

Fig. 7. - Coefficient de transmission d'un résonateur à quartz de coupe $\mathrm{Y}(\lambda=28,6 \mu \mathrm{m})$.

[Transmission coefficient of a $\mathrm{Y}$ cut quartz resonator $(\lambda=28.6 \mu \mathrm{m})$.

\section{Réflexion d'une onde de Rayleigh sous un sillon peu profond.}

Le problème de la réflexion d'une onde de Rayleigh sous un sillon gravé peu profond n'a été résolu par des méthodes analytiques que dans le cas des matériaux isotropes ou à symétrie trigonale [15-17]. La transposition aux milieux anisotropes était alors obtenue à partir de considérations empiriques $[18,19]$ ou à partir d'une approche par modes normaux [20].

Le schéma de principe d'un sillon peu profond est représenté figure 8 . L'onde se propageant à la surface

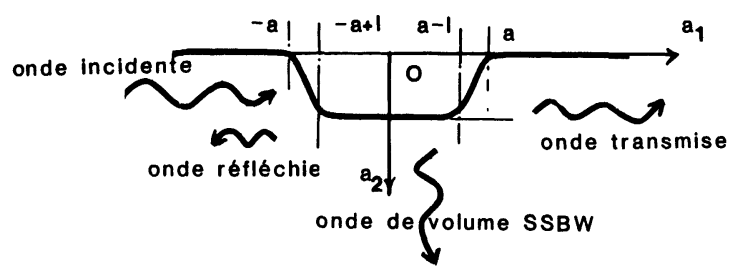

Fig. 8. - Schéma du sillon de réflexion pour l'onde de surface.

[Schematic of a reflexion groove for the surface wave.] du substrat gravé doit satisfaire à l'équation d'équilibre, ainsi qu'à la condition d'annulation de la contrainte normale sur la surface d'équation $a_{2}=$ $h f\left(a_{1}\right)$. Or l'onde de Rayleigh incidente $\stackrel{0}{u}_{j}$ ne peut pas remplir cette condition. Pour résoudre ce problème on utilise une méthode de perturbation en développant le déplacement mécanique [18] par approximations successives :

$$
u_{j}=\stackrel{0}{u}_{j}+\varepsilon \dot{1}_{j}+0\left(\varepsilon^{2}\right),
$$

où $\varepsilon$ est un infiniment petit.

En effectuant un développement en série de Taylor autour de la surface plane $a_{2}=0$, on obtient le terme correctif $\stackrel{1}{u_{j}}$ solution du système :

$$
\begin{gathered}
\rho_{0} \omega^{2} \stackrel{1}{u}_{j}-C_{i j k l} \stackrel{1}{u}_{k, l i}=0 \\
C_{2 j k l} \stackrel{1}{u}_{k, l}=\frac{h}{\varepsilon}\left(\frac{\partial f}{\partial a_{1}} C_{1 j k l} \stackrel{0}{u_{k, l}}-h f\left(a_{1}\right) C_{2 j k l} \stackrel{0}{u}, l l 2^{2}\right) .
\end{gathered}
$$

Comme précédemment en partie 1 , ce système est résolu en prenant la transformation de Fourier $\stackrel{\hat{u}}{u_{j}}$ spatiale du déplacement mécanique, où :

$$
\hat{\hat{u}}_{j}\left(k, a_{2}\right)=\sum_{r=1}^{3} C_{r} a_{j}^{(r)} \mathrm{e}^{-i k n n_{2}^{(r)} a_{2}} .
$$

Les poids $C_{r}$ de chaque terme de pénétration sont obtenus en utilisant la transformée de Fourier de la condition aux limites (18).

Les déplacements $\stackrel{1}{u}_{j}$ s'obtiennent par transformée de Fourier inverse :

$$
\begin{aligned}
& \stackrel{1}{u}_{j}\left(a_{1}, a_{2}\right)=\frac{1}{\sqrt{2 \pi}} \int_{-\infty}^{+\infty} \frac{D_{r}}{\Delta} a_{k}^{(r)} \mathrm{e}^{-i k n{ }^{(r)} a_{2}} \hat{H}(k, 0) \frac{h}{\varepsilon} \times \\
& \times \mathrm{e}^{-i k a_{1}} \mathrm{~d} k,
\end{aligned}
$$

où $D_{r}, a_{k}^{(r)}$ et $\Delta$ sont fonction du nombre d'onde d'intégration $k$ et où on a posé :

$$
\hat{H}(k, 0)=\frac{1}{\sqrt{2 \pi}} \int_{-\infty}^{+\infty} f\left(a_{1}\right) \mathrm{e}^{i\left(k-k_{\mathrm{R}}\right) a_{1}} \mathrm{~d} a_{1} .
$$

La constante $k_{\mathrm{R}}=\omega / V_{\mathrm{R}}$ est le nombre d'onde de Rayleigh.

Comme pour l'intégrale (8) qui a une forme similaire, on effectue le calcul de (20) dans le plan complexe des nombres d'onde en utilisant le contour d'intégration représenté figure 2. Deux types d'onde sont émises; d'abord les ondes de Rayleigh réfléchies et transmises correspondant à la contribution des pôles $k=-k_{\mathrm{R}}$ et $k=k_{\mathrm{R}}$; puis les ondes SSBW $[15,21]$ qui sont calculées en prenant la contribution des points critiques.

Nous ne nous intéressons ici qu'à l'onde de Rayleigh réfléchie. Le calcul du résidu du pôle $-k_{\mathrm{R}}$ nous 
permet d'obtenir l'amplitude normalisée de cette onde :

$$
U_{\mathrm{ref}}=-i \frac{\lambda_{\mathrm{R}}}{(\mathrm{d} \Delta / \mathrm{d} k)_{k=-k_{\mathrm{R}}}} D_{1} \hat{H}\left(-k_{\mathrm{R}}, 0\right) a_{1}^{(1)}
$$

avec

$$
a_{1}^{(1)}=1 \mathrm{~m}^{2} .
$$

Dans le cas d'un sillon rectangulaire de largeur $2 a$, on a $H\left(-k_{\mathrm{R}}, 0\right)=\frac{\sin 2 k_{\mathrm{R}} a}{2 k_{\mathrm{R}}} \cdot \frac{1}{\sqrt{2 \pi}}$. Le coefficient de réflexion $R$ (rapport des amplitudes de l'onde réfléchie et de l'onde incidente) est maximal pour $\sin 2 k_{\mathrm{R}} a=1$; on obtient alors en posant $h=\varepsilon \lambda$ :

$$
R=\frac{h}{\lambda} \frac{i \lambda}{V_{\mathrm{R}}(\mathrm{d} \Delta / \mathrm{d} V)_{V=-V_{\mathrm{R}}}} D_{1} a_{1}^{(1)} / U_{\mathrm{inc}},
$$

où $U_{\text {inc }}$ désigne l'amplitude de l'onde incidente.

3. 1 RÉSULTATS. - Les résultats numériques obtenus sont en bon accord avec les valeurs expérimentales existantes. Pour le cas d'un substrat de quartz de coupe ST, le coefficient de réflexion calculé est $R=$ $0,57 h / \lambda$ en bon accord avec la mesure [22] : $R=$ $0,54 h / \lambda$.

La méthode précédente a été également généralisée au cas des matériaux à fort couplage piézoélectrique. La valeur théorique obtenue pour un matériau de niobate de lithium de coupe Y.Z est $R=0,64 h / \lambda$, tandis que l'expérience fournit la valeur $[22,23]$ $R=0,67 h / \lambda$.

Sur la figure 9, nous avons représenté le coefficient de réflexion dans le cas d'un matériau de quartz en fonction de l'angle de rotation $\theta$ autour de l'axe $\mathrm{O} a_{1}$. La courbe en pointillés représente le coefficient de couplage électromécanique obtenu à l'aide des calculs exposés en deuxième partie [7].

Les calculs seront étendus ultérieurement à la recherche des ondes SSBW émises au niveau du sillon. De plus, une méthode de perturbation par échelles multiples est en cours d'élaboration pour examiner la réflexion de l'onde de Rayleigh sous un réseau comprenant un très grand nombre de sillons, ce qui représente le cas concret des résonateurs.

\section{Conclusion.}

Nous venons d'étudier les ondes émises dans un demi-espace anisotrope par application d'une excitation harmonique en tout point du milieu. Pour

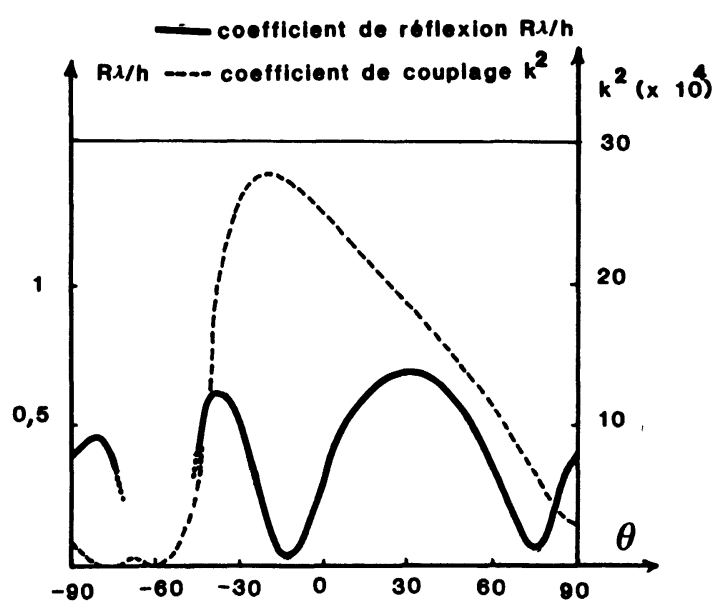

Fig. 9. - Coefficient de réflexion $R \lambda / h$ en fonction de l'angle de coupe $\theta$ autour de l'axe $\mathrm{O} a_{1}$ d'un substrat de quartz de coupe Y (courbes en traits pleins) et coefficient de couplage piézoélectrique (courbes en pointillés).

[Reflexion coefficient $R \lambda / h$ as a function of the cut angle $\theta$ around the $\mathrm{O} a_{1}$ axis of a $\mathrm{Y}$ cut quartz substrate (full curves) and electromechanical coupling coefficient (dotted curves).]

démontrer la validité de l'approche, nous nous sommes intéressés à deux types d'excitation :

- l'excitation due au potentiel électrostatique créé par le transducteur d'entrée déposé sur un milieu piézoélectrique,

- puis l'excitation due à un défaut de planéité de la surface, ici un sillon gravé faiblement profond.

Cette méthode est évidemment extensible à une multitude de phénomènes, la seule restriction étant que l'excitation doit être découplée du phénomène de propagation.

Nous avons montré que dans chaque cas étaient émises non seulement une onde de type Rayleigh correspondant à une solution de la partie homogène des équations de propagation et de la condition aux limites, mais également 3 ondes de volume SSBW.

Les applications numériques conduites dans le cas d'un milieu très anisotrope tel que le quartz sont en excellent accord avec les mesures effectuées et devraient conduire à une amélioration des dispositifs existants, filtres, résonateurs, ...

Une extension de ce travail à l'étude du régime transitoire est envisagée; les méthodes mises en œuvre par Cagniard et de Hoop [13] semblent être une excellente plate-forme de départ. 


\section{Bibliographie}

[1] White, R. M., Proc. IEEE 58 (8) (1970) 1238.

[2] LEWIS, M., IEEE Ultrasonics Symposium Proceedings (1977) 744.

[3] LeE, D. L., IEEE Trans. Sonics Ultrasonics SU-27, 1 (1980) 22.

[4] Joshi, S. G. and White, R. M., J. Acoust. Soc. Am. 46 (1969) 17.

[5] Ganguly, A. K. and Vassel, M. O., J. Appl. Phys. 44 (3) (1973) 1072.

[6] Milson, R. F., Reilly, N. H. C. and Redwood, M., IEEE Trans. on Sonics and Ultrasonics SU-24, 3 (1977) 147.

[7] Planat, M., Schiavone, P., J. Appl. Phys. 57 (1) (1985) 49.

[8] Lamb, H., Philos. Trans. R. Soc. London A 203 (1904) 1.

[9] Engan, H., IEEE Trans. Electron Devices ED 16 (1969) 1014.

[10] Lim, T. C. and Farnell, G. W., J. Acoust. Soc. Am. 45 (1969) 845.

[11] Morgan, D. P., IEEE Trans. Sonics Ultrasonics SU27, 3 (1980) 111.

[12] Ken'ICHIRo YASHIRo and NaOHISA Goto, IEEE Trans. Sonics Ultrasonics SU-25, 3 (1978) 146.
[13] Dieulesaint, E. et Royer, D., Ondes élastiques dans les solides (Masson et Cie, Paris) 1974.

[14] LEE, D. L., IEEE Ultrasonics Symposium Proceedings (1978) 675.

[15] Parekh, J. P. and Tuan, H. S., J. Appl. Phvs. 48 (3) (1977) 994.

[16] Simmons, D. A., J. Acoust. Soc. Am. 63 (5) (1978) 1292.

[17] Wu, S. D. and Tuan, H. S., J. Appl. Phys. 50 (1) (1979) 73.

[18] Tuan, H. S. and Li, R. C. M., J. Acoust. Soc. Am. 55 (6) (1974).

[19] Otto, O. W. and Gerard, H. M., IEEE Ultrasonics Symposium Proceedings (1977) 596.

[20] Datta, S. and Hunsinger, B. J., J. Appl. Phys. 50 (9) (1979) 5661.

[21] Ronnekleiv, A. and Souquet, J., J. Appl. Phys. 47 (10) (1976) 4422.

[22] MeYer, P. C. and Schultz, M. B., IEEE Ultrasonics Symposium Proceedings (1973) 500.

[23] Williamson, R. C. and Smith, H. I., IEEE Trans. Microwave Theory Techn. MTT 21 (4) (1979) 195. 